PROCEEDINGS OF THE

AMERICAN MATHEMATICAL SOCIETY

Volume 129 , Number 6, Pages 1861-1872

S 0002-9939(00)05966-9

Article electronically published on December 7, 2000

\title{
SUBDIVISION SCHEMES FOR ITERATED FUNCTION SYSTEMS
}

\author{
CHARLES A. MICCHELlI, THOMAS SAUER, AND YUESHENG XU
}

(Communicated by David R. Larson)

\begin{abstract}
We identify iterated function systems $\Phi$ and regular Borel measures $\mu$ such that the matrix subdivision process relative to a finite family $\mathcal{A}$ converges if and only if $\mathcal{A}$ satisfies certain spectral properties.
\end{abstract}

\section{IntRoduCtion}

Given any finite family of contractive mappings $\Phi$ on a metric space $X$ there is a unique invariant subset $\Omega$ of $X$ which is the union of all of its images under the contractive mappings. We suppose that there is a regular Borel measure $\mu$ on $\Omega$ such that the images of $\Omega$ under the contractive mappings partition $\Omega$ relative to $\mu$. Repeated iterations of the contractions lead to a sequence of finer partitions of $\Omega$. Moreover, the contractions partition any of the cells obtained at iteration level $r$ into subcells of level $r+1$. This refinement structure on $\Omega$ can be used to create vector fields on $\Omega$ with range in $\mathbb{R}^{n}$ in the following way. To this end, we require a family $\mathcal{A}$ of $n \times n$ matrices of the same cardinality as $\Phi$. Each mapping in $\Phi$ is associated with a unique matrix in $\mathcal{A}$. We start with a constant vector field $\boldsymbol{h}$ on $\Omega$ and break it into a piecewise constant function whose value on the cell $\phi(\Omega)$ is $\boldsymbol{A} \boldsymbol{h}$ where $\phi \in \Phi$ and $\boldsymbol{A} \in \mathcal{A}$ are associated. We iterate this procedure to get a sequence of piecewise constant functions on finer and finer partitions of $\Omega$. This process is referred to as matrix subdivision and is important in both geometric modeling and wavelet analysis [1, 4, 5, 6, 7, 8. If it converges, it produces a refinable function relative to $\mathcal{A}$ and $\Phi$. Our purpose here is to study it in greater generality than previously available. Our goal is to identify conditions on $\Phi$ and the measure $\mu$ such that a useful characterization of the convergence of the subdivision process to a function in $L_{p}(\Omega, \mu)$ can be given in terms of the spectral properties of $\mathcal{A}$.

Received by the editors September 27, 1999.

2000 Mathematics Subject Classification. Primary 54C40, 14E20; Secondary 46E25, 20C20.

Key words and phrases. Subdivision, iterated function systems, spectral radius.

The first author was supported by National Science Foundation under grants DMS-9504780 and DMS-9973427, by the Alexander von Humboldt foundation and by the Wavelets Strategic Research Programme, National University of Singapore, under a grant from the National Science and Technology Board and the Ministry of Education, Singapore.

The second author was supported by Deutsche Forschungsgemeinschaft with a Heisenberg fellowship, Sa $627 / 6$.

The third author was supported by National Science Foundation under grants DMS-9504780 and DMS-9973427, by the Alexander von Humboldt Foundation and by the "One Hundred Outstanding Young Chinese Scientists" program of the Chinese Academy of Sciences. 
This paper is organized into four sections. Section 2 is devoted to a description of subdivision schemes and the definition of convergence. A few simple observations are also made on convergent subdivision schemes. In Section 3 we define and discuss the concept of $\mu$-uniformity of a family of contractions for any $\mu$-measurable set. Finally, in Section 4, we establish a characterization of convergent subdivision schemes and prove that the subdivision scheme converges exponentially fast and that its limit function enjoys Hölder regularity.

\section{Contractions And subdivision}

For any integer $n>0$ we denote the set $\{0,1, \ldots, n-1\}$ by $\mathbb{Z}_{n}$ and use $\mathbb{Z}_{\infty}=$ $\{0,1, \ldots\}$. Let $X$ be a complete metric space, and let $\Phi=\left\{\phi_{\ell}: \ell \in \mathbb{Z}_{m}\right\}$ be a finite set of contractions on $X$. Recall the following facts from [2]. There exists a unique closed and bounded set $\Omega$ such that

$$
\Omega=\bigcup_{\ell \in \mathbb{Z}_{m}} \phi_{\ell}(\Omega)
$$

To any vector $\boldsymbol{j}=\left(j_{k}: k \in \mathbb{Z}_{r}\right) \in \mathbb{Z}_{m}^{r}$, that is, $j_{k} \in \mathbb{Z}_{m}, k \in \mathbb{Z}_{r}$, we associate the composite mapping

$$
\phi_{j}=\phi_{j_{0}} \circ \cdots \circ \phi_{j_{r-1}}
$$

and observe that

$$
\Omega=\bigcup_{j \in \mathbb{Z}_{m}^{r}} \phi_{j}(\Omega) .
$$

Also, for any nonempty set $\Gamma \subseteq X$ we define the set-valued mapping

$$
\mathcal{S}(\Gamma)=\bigcup_{\ell \in \mathbb{Z}_{m}} \phi_{\ell}(\Gamma)
$$

and, for $r \in \mathbb{Z}_{\infty}$, its iterates $\mathcal{S}^{r+1}(\Gamma)=\mathcal{S}\left(\mathcal{S}^{r}(\Gamma)\right)$. It is known that

$$
\lim _{r \rightarrow \infty} \mathcal{S}^{r}(\Gamma)=\Omega
$$

in the Hausdorff metric [2].

Let $x_{\boldsymbol{j}} \in X, \boldsymbol{j} \in \mathbb{Z}_{m}^{r}, r \in \mathbb{Z}_{\infty}$, be the fixed point of the contraction $\phi_{\boldsymbol{j}}$. It is proved in [2] that $\Omega$ is compact and is the closure of the set

$$
F_{\Phi}=\left\{x_{j}: j \in \mathbb{Z}_{m}^{r}, r \in \mathbb{Z}_{\infty}\right\}
$$

For every (infinite) vector $j=\left(j_{\ell}: \ell \in \mathbb{Z}_{\infty}\right) \in \mathbb{Z}_{m}^{\infty}$ and $r \in \mathbb{Z}_{\infty}$ we form the (finite) vectors $\boldsymbol{j}^{r}=\left(j_{\ell}: \ell \in \mathbb{Z}_{r}\right)$ and recall that $\lim _{r \rightarrow \infty} x_{\boldsymbol{j}^{r}}$ exists. We shall denote this limit by $x_{\boldsymbol{j}}$. Moreover, for every $x, y \in \Omega$ there exists a $\boldsymbol{j} \in \mathbb{Z}_{m}^{\infty}$ such that $x=$ $\lim _{r \rightarrow \infty} \phi_{\boldsymbol{j}^{r}}(y)$.

Suppose that $\mu$ is a regular Borel measure on $\Omega$ with $0<\mu(\Omega)<\infty$; cf. 9 . For $\Gamma \subseteq \Omega$, we denote by $L_{p}(\Gamma, \mu), 1 \leq p<\infty$, the spaces of $p$-integrable functions $f: \Gamma \rightarrow \mathbb{R}$ for which

$$
\|f\|_{L_{p}(\Gamma, \mu)}:=\left(\int_{\Gamma}|f(x)|^{p} d \mu(x)\right)^{1 / p}
$$

is finite. Mostly, we will write $\|f\|_{L_{p}(\Gamma)}$ and in the case that $\Gamma=\Omega$ use $\|f\|_{p}$. Moreover, we will use $L_{p}^{s \times t}(\Gamma, \mu)$ for all $\mathbb{R}^{s \times t}$ matrix-valued functions on $\Gamma$, that 
is, $\boldsymbol{F}=\left(f_{\ell k}: \ell \in \mathbb{Z}_{s}, k \in \mathbb{Z}_{t}\right)$, such that $f_{\ell k} \in L_{p}(\Gamma, \mu), \ell \in \mathbb{Z}_{s}, k \in \mathbb{Z}_{t}$, and equip it with the norm

$$
\|\boldsymbol{F}\|_{L_{p}^{s \times t}(\Gamma, \mu)}=\sum_{\ell \in \mathbb{Z}_{s}} \sum_{k \in \mathbb{Z}_{t}}\left\|f_{\ell k}\right\|_{L_{p}(\Gamma, \mu)} .
$$

As above, we use the abbreviated notation $\|\boldsymbol{F}\|_{L_{p}(\Gamma)},\|\boldsymbol{F}\|_{p}$ and also $L_{p}^{s}(\Gamma, \mu)$ for the space $L_{p}^{s \times 1}(\Gamma, \mu)$. In this way, the norm of a vector field $\boldsymbol{f}=\left(f_{j}: j \in \mathbb{Z}_{s}\right) \in$ $L_{p}^{s}(\Gamma, \mu)$ is defined as the norm of $\boldsymbol{f}$, interpreted as an $s \times 1$ matrix-valued function. For any two matrices $\boldsymbol{A} \in \mathbb{R}^{n \times s}, \boldsymbol{B} \in \mathbb{R}^{t \times n}$ it follows from Minkowski's inequality that

where

$$
\|\boldsymbol{A F} \boldsymbol{B}\|_{L_{p}^{n \times n}(\Gamma)} \leq\left\|\boldsymbol{A}^{T}\right\|\|\boldsymbol{B}\|\|\boldsymbol{F}\|_{L_{p}^{s \times t}(\Gamma)}
$$

$$
\|\boldsymbol{A}\|:=\max _{j \in \mathbb{Z}_{n}} \sum_{k \in \mathbb{Z}_{s}}\left|\boldsymbol{A}_{j k}\right|
$$

We assume that the measure $\mu$ and the family $\Phi$ of contractive mappings have the following properties. There exist positive constants $C, D$ such that for all $\ell \in \mathbb{Z}_{m}$, $\Gamma \subseteq \Omega$ and $f \in L_{p}(\Gamma, \mu)$

$$
C\|f\|_{L_{p}\left(\phi_{\ell}(\Gamma), \mu\right)} \leq\left\|f \circ \phi_{\ell}\right\|_{L_{p}(\Gamma, \mu)} \leq D\|f\|_{L_{p}\left(\phi_{\ell}(\Gamma), \mu\right)} .
$$

Moreover, we require that

$$
\phi_{\ell} \text { is injective, } \quad \ell \in \mathbb{Z}_{m},
$$

and

$$
\mu\left(\phi_{\ell}(\Omega) \cap \phi_{k}(\Omega)\right)=0, \quad \ell, k \in \mathbb{Z}_{m}, \ell \neq k .
$$

For $\Gamma \subseteq \Omega$ and $\ell \in \mathbb{Z}_{m}$, equation (2.6) implies that

$$
C^{p} \mu\left(\phi_{\ell}(\Gamma)\right) \leq \mu(\Gamma) \leq D^{p} \mu\left(\phi_{\ell}(\Gamma)\right) .
$$

In particular, we obtain that $\mu\left(\phi_{\boldsymbol{j}}(\Omega)\right)>0, \boldsymbol{j} \in \mathbb{Z}_{m}^{r}, r \in \mathbb{Z}_{\infty}$. Moreover, from (2.6) and (2.8) it follows that

$$
\mu\left(\phi_{\boldsymbol{j}}(\Omega) \cap \phi_{\boldsymbol{k}}(\Omega)\right)=0, \quad \boldsymbol{j}, \boldsymbol{k} \in \mathbb{Z}_{m}^{r}, \boldsymbol{j} \neq \boldsymbol{k}, \quad r \in \mathbb{Z}_{\infty} .
$$

We shall make use of the family of pushforward measures $\left\{\mu_{\boldsymbol{j}}: \boldsymbol{j} \in \mathbb{Z}_{m}^{r}, r \in \mathbb{Z}_{\infty}\right\}$ defined by setting, for any $\mu$-measurable set $\Gamma \subseteq \Omega$,

$$
\mu_{\boldsymbol{j}}(\Gamma)=\mu\left(\phi_{\boldsymbol{j}}(\Gamma)\right) .
$$

Since for all $f \in L_{p}(\Omega)$ we have that

$$
\left\|f \circ \phi_{\boldsymbol{j}}\right\|_{L_{p}\left(\Gamma, \mu_{j}\right)}=\|f\|_{L_{p}\left(\phi_{\boldsymbol{j}}(\Gamma), \mu\right)}
$$

condition (2.6) says, for $\ell \in \mathbb{Z}_{m}$, that on the set $\phi_{\ell}(\Omega)$ the $L_{p}(\Gamma, \mu)$ and $L_{p}\left(\phi_{\ell}(\Gamma), \mu_{\ell}\right)$ norms are equivalent.

Let $\mathcal{A}=\left\{\boldsymbol{A}_{\ell}: \ell \in \mathbb{Z}_{m}\right\}$ be a finite collection of $n \times n$ matrices. For every $\boldsymbol{j}=$ $\left(j_{k}: k \in \mathbb{Z}_{r}\right) \in \mathbb{Z}_{m}^{r}$ and $r \in \mathbb{Z}_{\infty}$, we define the matrices $\boldsymbol{A}_{\boldsymbol{j}}=\boldsymbol{A}_{j_{r-1}} \cdots \boldsymbol{A}_{j_{0}}$ and the piecewise constant matrix-valued function $\boldsymbol{A}^{[r]} \in L_{p}^{n \times n}(\Omega)$

$$
\boldsymbol{A}^{[r]}=\sum_{\boldsymbol{j} \in \mathbb{Z}_{m}^{r}} \boldsymbol{A}_{\boldsymbol{j}} \chi_{\phi_{\boldsymbol{j}}(\Omega)}, \quad r \in \mathbb{Z}_{\infty}
$$

where, for $\Gamma \subseteq \Omega$, we write $\chi_{\Gamma}$ for the characteristic function of $\Gamma$. Note that the order of the matrices in the product (2.10) is the reverse of the order of the 
mappings in the composition (2.2). From the upper bound in equation (2.6) and the injectivity of $\phi_{\ell}, \ell \in \mathbb{Z}_{m}$, it follows, for $r \in \mathbb{Z}_{\infty}$ and $k \in \mathbb{Z}_{m}$, that

$$
\boldsymbol{A}^{[r+1]} \circ \phi_{k}=\boldsymbol{A}^{[r]} \boldsymbol{A}_{k}, \quad \mu \text {-a.e. }
$$

Given any two vectors $\boldsymbol{a}, \boldsymbol{b} \in \mathbb{R}^{n}$ we denote by $\boldsymbol{a} \otimes \boldsymbol{b} \in \mathbb{R}^{n \times n}$ their tensor product

$$
\boldsymbol{a} \otimes \boldsymbol{b}=\left(a_{\ell} b_{k}: \ell, k \in \mathbb{Z}_{n}\right)
$$

and use $\mathbf{1}$ for the vector in $\mathbb{R}^{n}$ whose entries are all 1 .

Definition 2.1. A finite collection $\mathcal{A}$ of $n \times n$ matrices admits a $L_{p}(\Omega, \mu)$ convergent subdivision scheme if there exists a nontrivial vector field $\boldsymbol{f} \in L_{p}^{n}(\Omega, \mu)$ such that

$$
\lim _{r \rightarrow \infty} A^{[r]}=\mathbf{1} \otimes f
$$

Whenever the subdivision scheme converges we call $f$ its limit.

Later, we shall use the fact that the set of matrices

$$
\boldsymbol{A}_{\ell}:=\boldsymbol{H}:=\frac{1}{n} \mathbf{1} \otimes \mathbf{1}, \quad \ell \in \mathbb{Z}_{m}
$$

admit an $L_{p}(\Omega, \mu)$ convergent subdivision scheme with limit $\boldsymbol{h}:=n^{-1} \mathbf{1}$.

Proposition 2.2. If $\mathcal{A}$ admits a convergent subdivision scheme with limit $\boldsymbol{f}$, then, for every $\ell \in \mathbb{Z}_{m}$, we have that

$$
\boldsymbol{f} \circ \phi_{\ell}=\boldsymbol{A}_{\ell}^{T} \boldsymbol{f}, \quad \mu-a . e .
$$

Proof. We fix $\ell \in \mathbb{Z}_{m}$. The inequalities

$$
\begin{aligned}
\| & \mathbf{1} \otimes \boldsymbol{f} \circ \phi_{\ell}-\mathbf{1} \otimes\left(\boldsymbol{A}^{T} \boldsymbol{f}\right)\left\|_{p}=\right\| \mathbf{1} \otimes \boldsymbol{f} \circ \phi_{\ell}-(\mathbf{1} \otimes \boldsymbol{f}) \boldsymbol{A}_{\ell} \|_{p} \\
& \leq\left\|\mathbf{1} \otimes \boldsymbol{f} \circ \phi_{\ell}-\boldsymbol{A}^{[r+1]} \circ \phi_{\ell}\right\|_{p}+\left\|\left(\mathbf{1} \otimes \boldsymbol{f}-\boldsymbol{A}^{[r]}\right) \boldsymbol{A}_{\ell}\right\|_{p} \\
& \leq D\left\|\mathbf{1} \otimes \boldsymbol{f}-\boldsymbol{A}^{[r+1]}\right\|_{p}+\left\|\boldsymbol{A}_{\ell}\right\|\left\|\mathbf{1} \otimes \boldsymbol{f}-\boldsymbol{A}^{[r]}\right\|_{p}
\end{aligned}
$$

yield the result.

We call any solution of equation (2.13) a refinable vector field associated to the refinement matrices $\mathcal{A}$. Therefore, the limit of a convergent subdivision scheme is always a refinable vector field.

We say that the measure $\mu$ is strictly positive on $\Omega$ if, for all $x \in \Omega$ and $\delta>0$, the measure of the ball $B(x ; \delta)=\{y: y \in \Omega, d(x, y) \leq \delta\}$ is strictly positive, that is, $\mu(B(x ; \delta))>0$. Observe that a strictly positive measure $\mu$ satisfies, for any continuous function $f \in C(\Omega)$ and $x \in \Omega$, the inequality

$$
\left|\frac{1}{\mu(B(x ; \delta))} \int_{B(x ; \delta)} f(y) d \mu(y)-f(x)\right| \leq \omega_{\infty}(f ; \delta),
$$

where $\omega_{\infty}$ denotes the modulus of continuity of $f$ given by

$$
\omega_{\infty}(f ; \delta)=\max \{|f(x)-f(y)|: x, y \in \Omega, d(x, y)<\delta\} .
$$

Since $\lim _{\delta \rightarrow 0} \omega_{\infty}(f ; \delta)=0$ for any $f \in C(\Omega)$ we conclude that whenever $f \in C(\Omega)$ vanishes $\mu$-a.e. on $\Omega$ it must be identically zero on $\Omega$. This fact leads us to the next result. 
Corollary 2.3. Suppose that $\mu$ is strictly positive on $\Omega$ and that $\mathcal{A}$ admits a convergent subdivision scheme with limit $\boldsymbol{f}$. If $\boldsymbol{f}$ is continuous on $\Omega$, then, for every $x \in \Omega$,

$$
\boldsymbol{f}(x) \neq 0 .
$$

Proof. Since $\boldsymbol{f}$ and $\phi_{\ell}, \ell \in \mathbb{Z}_{m}$, are continuous we have, for any $y \in \Omega$ and $\boldsymbol{j} \in \mathbb{Z}_{m}^{r}$, $r \in \mathbb{Z}_{\infty}$, that

$$
\boldsymbol{f}\left(\phi_{\boldsymbol{j}}(y)\right)=\boldsymbol{A}_{\boldsymbol{j}}^{T} \boldsymbol{f}(y) .
$$

Suppose that there exists a $y \in \Omega$ such that $\boldsymbol{f}(y)=0$. For any $x \in \Omega$ we choose a sequence $\boldsymbol{j}^{r} \in \mathbb{Z}_{m}^{r}, r \in \mathbb{Z}_{\infty}$, such that $\lim _{r \rightarrow \infty} \phi_{\boldsymbol{j}^{r}}(y)=x$. Since $\boldsymbol{f}(y)=0$ equation (2.14) and the continuity of $\boldsymbol{f}$ imply that

$$
\boldsymbol{f}(x)=\lim _{r \rightarrow \infty} \boldsymbol{f}\left(\phi_{\boldsymbol{j}^{r}}(y)\right)=\boldsymbol{A}_{\boldsymbol{j}^{r}}^{T} \boldsymbol{f}(y)=0,
$$

which violates the assumption that $\mathcal{A}$ admits a convergent subdivision scheme. This contradiction proves the result.

Corollary 2.4. Suppose that $\mathcal{A}$ admits a convergent subdivision scheme with limit $\boldsymbol{f}$. If at least two of the matrices in $\mathcal{A}$ are nonsingular, then $\boldsymbol{f} \neq 0, \mu$-a.e.

Proof. Let $\boldsymbol{A}_{\ell}$ and $\boldsymbol{A}_{k}, \ell \neq k \in \mathbb{Z}_{m}$, be two nonsingular matrices in $\mathcal{A}$ and set $\Gamma=\{x \in \Omega: \boldsymbol{f}(x)=0\}$. From the refinement equation (2.13) and the invertibility of $\boldsymbol{A}_{\ell}$ and $\boldsymbol{A}_{k}$ we conclude that

$$
\Gamma=\phi_{\ell}(\Gamma)=\phi_{k}(\Gamma)
$$

and therefore, by (2.8) we obtain

$$
\mu(\Gamma) \leq \mu\left(\phi_{\ell}(\Gamma) \cap \phi_{k}(\Gamma)\right) \leq \mu\left(\phi_{\ell}(\Omega) \cap \phi_{k}(\Omega)\right)=0 .
$$

\section{3. $\mu$-UNIFORMITY}

For $\ell \in \mathbb{Z}_{m}$ we define the set

$$
\Omega_{\ell}^{r}=\bigcup\left\{\phi_{j}(\Omega): j=\left(j_{k}: k \in \mathbb{Z}_{r+1}\right) \in \mathbb{Z}_{m}^{r+1}, j_{r}=\ell\right\}
$$

and note that

$$
\Omega=\bigcup_{\ell \in \mathbb{Z}_{m}} \Omega_{\ell}^{r}
$$

From (2.4) it follows that

$$
\lim _{r \rightarrow \infty} \Omega_{\ell}^{r}=\Omega, \quad \ell \in \mathbb{Z}_{m}
$$

in the Hausdorff metric on $X$. We require an additional property.

Definition 3.1. We say that the family $\Phi$ of contractions is $\mu$-uniform if for any $\mu$-measurable set $\Gamma \subseteq \Omega, \mu(\Gamma)>0$ and $\ell \in \mathbb{Z}_{m}$ the condition

$$
\liminf _{r \rightarrow \infty} \mu\left(\Gamma \cap \Omega_{\ell}^{r}\right)>0
$$

is satisfied.

Let us first provide sufficient conditions on the family of contractions $\Phi$ and the measure $\mu$ so that $\Phi$ is $\mu$-uniform. 
Lemma 3.2. If there exist positive constants $\rho_{\ell}, \ell \in \mathbb{Z}_{m}$, such that $\mu_{\ell}=\rho_{\ell} \mu$, $\ell \in \mathbb{Z}_{m}$, then, for any $f \in L_{p}(\Omega)$ and $\ell \in \mathbb{Z}_{m}$, we have that

$$
\lim _{r \rightarrow \infty}\|f\|_{L_{p}\left(\Omega_{\ell}^{r}\right)}=\rho_{\ell}^{1 / p}\|f\|_{L_{p}(\Omega)} .
$$

Choosing any $\mu$-measurable $\Gamma \subseteq \Omega$ and the function $f=\chi_{\Gamma}$ in (3.3) yields the following result.

Corollary 3.3. If there exist positive constants $\rho_{\ell}, \ell \in \mathbb{Z}_{m}$, such that $\mu_{\ell}=\rho_{\ell} \mu$, $\ell \in \mathbb{Z}_{m}$, then $\Phi$ is $\mu$-uniform.

A change of variables arguments implies the following result.

Corollary 3.4. If $X=\mathbb{R}^{s}, \Phi$ consists of affine mappings and $\mu$ is the Lebesgue measure on $\mathbb{R}^{s}$, then $\Phi$ is $\mu$-uniform.

To prove Lemma 3.2 we need another ancillary fact.

Lemma 3.5. For any $f \in C(\Omega)$ there exists a sequence $\varphi^{r}, r \in \mathbb{Z}_{\infty}$, of piecewise constant functions of the form

$$
\varphi^{r}=\sum_{\boldsymbol{j} \in \mathbb{Z}_{m}^{r}} c_{\boldsymbol{j}} \chi_{\phi_{\boldsymbol{j}}(\Omega)}, \quad c_{\boldsymbol{j}} \in \mathbb{R}, \quad \boldsymbol{j} \in \mathbb{Z}_{m}^{r},
$$

such that

$$
\lim _{r \rightarrow \infty}\left\|f-\varphi^{r}\right\|_{L_{p}(\Omega)}=0 .
$$

Proof. We choose some $y \in \Omega$ and set

$$
\varphi^{r}(f)=\sum_{\boldsymbol{j} \in \mathbb{Z}_{m}^{r}}\left(f \circ \phi_{j}\right)(y) \chi_{\phi_{j}(\Omega)} .
$$

Let $\delta_{r}=\max \left\{d(x, y): x, y \in \phi_{\boldsymbol{j}}(\Omega), \boldsymbol{j} \in \mathbb{Z}_{m}^{r}\right\}$ and note that, since $\Omega$ is bounded and $\Phi$ is a family of contractions, $\lim _{r \rightarrow \infty} \delta_{r}=0$. Note that, for any $r \in \mathbb{Z}_{\infty}$, we can write

$$
f(x)=\sum_{j \in \mathbb{Z}_{m}^{r}} f(x) \chi_{\phi_{\boldsymbol{j}}(\Omega)}(x), \quad x \in \Omega,
$$

and therefore

$$
\begin{aligned}
\left\|\varphi^{r}-f\right\|_{L_{p}(\Omega)} & =\left(\int_{\Omega}\left|\sum_{\boldsymbol{j} \in \mathbb{Z}_{m}^{r}}\left(\left(f \circ \phi_{j}\right)(y)-f(x)\right) \chi_{\phi_{\boldsymbol{j}}(\Omega)}(x)\right|^{p} d \mu(x)\right)^{1 / p} \\
& =\left(\sum_{\boldsymbol{j} \in \mathbb{Z}_{m}^{r}} \int_{\phi_{\boldsymbol{j}}(\Omega)}\left|\left(f \circ \phi_{\boldsymbol{j}}\right)(y)-f(x)\right|^{p} d \mu(x)\right)^{1 / p} \\
& \leq \omega_{\infty}\left(f ; \delta_{r}\right)(\mu(\Omega))^{1 / p} .
\end{aligned}
$$

Thus, the result follows.

Proof of Lemma 3.2. For $r \in \mathbb{Z}_{\infty}$ let $\varphi^{r}$ be a function of the form (3.3). Associated to any $\boldsymbol{j}=\left(j_{k}: k \in \mathbb{Z}_{r}\right)$ we introduce the constant $\rho_{\boldsymbol{j}}=\prod_{k \in \mathbb{Z}_{r}} \rho_{j_{k}}$ and note that $\rho_{j}=\frac{\mu_{j}(\Omega)}{\mu(\Omega)}$. For $\ell \in \mathbb{Z}_{m}$ we have that

$$
\left\|\varphi^{r}\right\|_{L_{p}\left(\Omega_{\ell}^{r}\right)}^{p}=\sum_{\boldsymbol{j} \in \mathbb{Z}_{m}^{r}}\left|c_{\boldsymbol{j}}\right|^{p} \rho_{\boldsymbol{j}} \rho_{\ell} \mu(\Omega)=\rho_{\ell} \sum_{\boldsymbol{j} \in \mathbb{Z}_{m}^{r}}\left|c_{\boldsymbol{j}}\right|^{p} \rho_{\boldsymbol{j}} \mu(\Omega)=\rho_{\ell}\left\|\varphi^{r}\right\|_{L_{p}(\Omega)}^{p} .
$$


Next, we choose $g \in C(\Omega)$. By Lemma 3.5, given any $\varepsilon>0$ there is an $r_{0} \in \mathbb{Z}_{\infty}$ such that for all $r>r_{0}$ we have that $\left\|\varphi^{r}(g)-g\right\|_{L_{p}(\Omega)}<\varepsilon / 2$ and therefore, since $\rho_{\ell} \leq 1, \ell \in \mathbb{Z}_{m}$,

$$
\begin{aligned}
\|g\|_{L_{p}\left(\Omega_{\ell}^{r}\right)} & \leq\left\|\varphi^{r}(g)\right\|_{L_{p}\left(\Omega_{\ell}^{r}\right)}+\left\|g-\varphi^{r}(g)\right\|_{L_{p}(\Omega)} \leq \rho_{\ell}^{1 / p}\left\|\varphi^{r}(g)\right\|_{L_{p}(\Omega)}+\frac{\varepsilon}{2} \\
& \leq \rho_{\ell}^{1 / p}\|g\|_{L_{p}(\Omega)}+\varepsilon .
\end{aligned}
$$

In exactly the same way we also obtain

$$
\|g\|_{L_{p}\left(\Omega_{\ell, r}\right)} \geq \rho_{\ell}^{1 / p}\|g\|_{L_{p}(\Omega)}-\varepsilon
$$

which proves (3.3) for continuous functions. For $f \in L_{p}(\Omega)$ we let $\varepsilon>0$ and pick $g \in C(\Omega)$ such that $\|f-g\|_{L_{p}(\Omega)}<\varepsilon / 3$. By what we just proved, we can also choose an $r_{0} \in \mathbb{Z}_{\infty}$ such that, for any $r>r_{0}$ and $\ell \in \mathbb{Z}_{m}$,

$$
\left|\|g\|_{L_{p}\left(\Omega_{\ell}^{r}\right)}-\rho_{\ell}^{1 / p}\|g\|_{L_{p}(\Omega)}\right| \leq \varepsilon / 3 .
$$

Hence, for $r>r_{0}$ and $\ell \in \mathbb{Z}_{m}$ we obtain

$$
\|f\|_{L_{p}\left(\Omega_{\ell}^{r}\right)} \leq\|g\|_{L_{p}\left(\Omega_{\ell}^{r}\right)}+\frac{\varepsilon}{3} \leq \rho_{\ell}^{1 / p}\|g\|_{L_{p}(\Omega)}+\frac{2}{3} \varepsilon \leq \rho_{\ell}^{1 / p}\|f\|_{L_{p}(\Omega)}+\varepsilon,
$$

and likewise

$$
\|f\|_{L_{p}\left(\Omega_{\ell}^{r}\right)} \geq \rho_{\ell}^{1 / p}\|g\|_{L_{p}(\Omega)}-\varepsilon
$$

which proves the lemma.

Based on property (3.2) we have the following result.

Lemma 3.6. If $\Phi$ is $\mu$-uniform in $\Omega$, then, for any $f \in L_{p}(\Omega)$ for which there exists $\ell \in \mathbb{Z}_{m}$ such that

$$
\lim _{r \rightarrow \infty}\|f\|_{L_{p}\left(\Omega_{\ell}^{r}\right)}=0
$$

it follows that $f=0, \mu-a . e$.

Proof. Let $f \in L_{p}(\Omega)$ satisfy (3.5) and suppose that $f \neq 0$. Then there exists a constant $\delta>0$ such that $\mu\left(F_{\delta}\right)>0$, where

$$
F_{\delta}:=\{x:|f(x)|>\delta\} .
$$

Therefore, we obtain

$$
\|f\|_{L_{p}\left(\Omega_{\ell}^{r}\right)} \geq \delta\left(\mu\left(F_{\delta} \cap \Omega_{\ell}^{r}\right)\right)^{1 / p} .
$$

Since $\Phi$ is $\mu$-uniform, this yields the contradiction

$$
0=\liminf _{r \rightarrow \infty}\|f\|_{L_{p}\left(\Omega_{\ell}^{r}\right)} \geq \delta \liminf _{r \rightarrow \infty}\left(\mu\left(F_{\delta} \cap \Omega_{\ell}^{r}\right)\right)^{1 / p}>0 .
$$




\section{Convergence of Subdivision SChemes}

Our first result in this section provides a necessary condition for the convergence of the matrix subdivision scheme.

Proposition 4.1. If $\Phi$ is $\mu$-uniform and $\mathcal{A}$ admits a convergent subdivision scheme, then

$$
\boldsymbol{A}_{\ell} \mathbf{1}=\mathbf{1}, \quad \ell \in \mathbb{Z}_{m}
$$

Proof. For any $j \in \mathbb{Z}_{m}^{r+1}$ and $\mu$-a.e. $x \in \phi_{\boldsymbol{j}}(\Omega)$ we conclude from the definition (2.10) that $\boldsymbol{A}^{[r+1]}(x)=\boldsymbol{A}_{\boldsymbol{j}}$. Hence, for $\ell \in \mathbb{Z}_{m}$ and $r \in \mathbb{Z}_{\infty}$ we get that

$$
\left.\boldsymbol{A}^{[r+1]}\right|_{\Omega_{\ell}^{r}}=\left.\boldsymbol{A}_{\ell} \boldsymbol{A}^{[r]}\right|_{\Omega_{\ell}^{r}}, \quad \mu \text {-a.e., }
$$

and consequently we obtain

$$
\begin{gathered}
\left\|\left(\mathbf{1}-\boldsymbol{A}_{\ell} \mathbf{1}\right) \otimes \boldsymbol{f}\right\|_{L_{p}\left(\Omega_{\ell}^{r}\right)}=\left\|\mathbf{1} \otimes \boldsymbol{f}-\boldsymbol{A}_{\ell}(\mathbf{1} \otimes \boldsymbol{f})\right\|_{L_{p}\left(\Omega_{\ell}^{r}\right)} \\
\leq\left\|\mathbf{1} \otimes \boldsymbol{f}-\boldsymbol{A}^{[r+1]}\right\|_{p}+\left\|\boldsymbol{A}^{[r+1]}-\boldsymbol{A}_{\ell}(\mathbf{1} \otimes \boldsymbol{f})\right\|_{L_{p}\left(\Omega_{\ell}^{r}\right)} \\
=\left\|\mathbf{1} \otimes \boldsymbol{f}-\boldsymbol{A}^{[r+1]}\right\|_{p}+\left\|\boldsymbol{A}_{\ell}\left(\boldsymbol{A}^{[r]}-\mathbf{1} \otimes \boldsymbol{f}\right)\right\|_{L_{p}\left(\Omega_{\ell}^{r}\right)} \\
\leq\left\|\mathbf{1} \otimes \boldsymbol{f}-\boldsymbol{A}^{[r+1]}\right\|_{p}+\left\|\boldsymbol{A}_{\ell}^{T}\right\|\left\|\mathbf{1} \otimes \boldsymbol{f}-\boldsymbol{A}^{[r]}\right\|_{p} .
\end{gathered}
$$

Since the upper bound in the last inequality converges to zero for $r \rightarrow \infty$, Lemma 3.6 proves the result.

To state the next result we use $\langle\cdot, \cdot\rangle$ for the standard inner product on $\mathbb{R}^{n}$.

Corollary 4.2. If the hypothesis of Proposition 4.1 is satisfied and $\boldsymbol{f}$ is the limit of the subdivision scheme $\mathcal{A}$, then

$$
\langle\boldsymbol{f}, \mathbf{1}\rangle=1, \quad \mu \text {-a.e. }
$$

Proof. By Proposition 4.1 for $r \in \mathbb{Z}_{\infty}, A^{[r]} \mathbf{1}=\mathbf{1}, \mu$-a.e., and therefore

$$
\mathbf{1}=\lim _{r \rightarrow \infty} \boldsymbol{A}^{[r]} \mathbf{1}=(\mathbf{1} \otimes \boldsymbol{f}) \mathbf{1}
$$

which proves equation (4.3).

For the next corollary we introduce, for $\mu$-a.e. $y \in \Omega, f \in L_{p}(\Omega)$ and $r \in \mathbb{Z}_{\infty}$, the following function on $\Omega$ which acts like a modulus of continuity,

$$
\omega_{r}(f)(y)=\left(\sum_{\boldsymbol{j} \in \mathbb{Z}_{m}^{r}} \int_{\phi_{\boldsymbol{j}}(\Omega)}\left|f(x)-\left(f \circ \phi_{\boldsymbol{j}}\right)(y)\right|^{p} d \mu(x)\right)^{1 / p},
$$

and extend it to vector fields $\boldsymbol{f}=\left(f_{k}: k \in \mathbb{Z}_{n}\right)$ by setting

$$
\omega_{r}(\boldsymbol{f})=\sum_{k \in \mathbb{Z}_{n}} \omega_{r}\left(f_{k}\right)
$$

Corollary 4.3. If $\mathcal{A}$ admits a convergent subdivision scheme with limit $\boldsymbol{f}$, then

$$
\lim _{r \rightarrow \infty} \omega_{r}(\boldsymbol{f})=0, \quad \mu-a . e .
$$


Proof. We have $\mu$-a.e. $y \in \Omega$ such that

$$
\omega_{r}(\boldsymbol{f})(y)=\left\|\boldsymbol{f}^{T}(y)\left(\mathbf{1} \otimes \boldsymbol{f}-\boldsymbol{A}^{[r]}\right)\right\|_{p}=\left\|\boldsymbol{f}-\left(\boldsymbol{A}^{[r]} \boldsymbol{f}\right)(y)\right\|_{p} .
$$

The first identity follows readily from the fact that $f$ is the limit function of a convergent subdivision scheme and thus satisfies (2.13) and (4.3). For the second identity we compute that

$$
\begin{aligned}
\left\|\boldsymbol{f}-\left(\boldsymbol{A}^{[r]} \boldsymbol{f}\right)(y)\right\|_{p} & =\sum_{k \in \mathbb{Z}_{n}}\left(\int_{\Omega}\left|f_{k}(x)-\left(\boldsymbol{A}^{[r]} \boldsymbol{f}\right)_{k}(y)\right|^{p} d \mu(x)\right)^{1 / p} \\
& =\sum_{k \in \mathbb{Z}_{n}}\left(\sum_{\boldsymbol{j} \in \mathbb{Z}_{m}^{r}} \int_{\phi_{\boldsymbol{j}}(\Omega)}\left|f_{k}(x)-\left(\boldsymbol{A}_{\boldsymbol{j}} \boldsymbol{f}\right)_{k}(y)\right|^{p} d \mu(x)\right)^{1 / p} \\
& =\omega_{r}(\boldsymbol{f})(y) .
\end{aligned}
$$

This fact is a consequence of Theorem 4.8 , however, we put it here since it has a partial converse which we state next.

Proposition 4.4. Suppose that $\boldsymbol{f} \in L_{p}(\Omega)$ is a nontrivial vector field which is refinable relative to a family $\mathcal{A}$ of matrices, which satisfies the equation $\langle\mathbf{1}, \boldsymbol{f}\rangle=1$ $\mu$-a.e. and the conditions

$$
\lim _{r \rightarrow \infty} \omega_{r}(\boldsymbol{f})=0, \quad \mu-a . e .
$$

and

$$
\mathbb{R}^{n}=\operatorname{span}\{\boldsymbol{f}(y): \mu \text {-a.e. } y \in \Omega\} .
$$

Then $\mathcal{A}$ admits a convergent subdivision scheme with limit $\boldsymbol{f}$.

Proof. This fact follows conveniently from equation (4.5).

Now we will characterize the convergence of matrix subdivision schemes. For this purpose we define the difference operator $\boldsymbol{D}: \mathbb{R}^{n} \rightarrow \mathbb{R}^{n-1}$ by setting, for any $\boldsymbol{c}=\left(c_{\ell}: \ell \in \mathbb{Z}_{n}\right)$,

$$
\boldsymbol{D} \boldsymbol{c}=\left(c_{\ell+1}-c_{\ell}: \ell \in \mathbb{Z}_{n-1}\right) .
$$

Its representation as an $(n-1) \times n$ matrix is

$$
\boldsymbol{D}=\left[\begin{array}{cccc}
-1 & 1 & & \\
& \ddots & \ddots & \\
& & -1 & 1
\end{array}\right]
$$

Observe the following fact.

Lemma 4.5. Let $\boldsymbol{A} \in \mathbb{R}^{n \times n}$.

1. $\boldsymbol{A} \mathbf{1}=\mathbf{0}$ if and only if there is a matrix $\boldsymbol{B} \in \mathbb{R}^{n \times(n-1)}$ such that $\boldsymbol{A}=\boldsymbol{B} \boldsymbol{D}$.

2. If $\boldsymbol{A} \mathbf{1}=\mathbf{1}$, then there is a matrix $\boldsymbol{B} \in \mathbb{R}^{(n-1) \times(n-1)}$ such that $\boldsymbol{D} \boldsymbol{A}=\boldsymbol{B} \boldsymbol{D}$.

We pattern the following notion after Jia [3]. 
Definition 4.6. Let $\mathcal{A}$ be a finite collection of $n \times n$ matrices of cardinality $m$. The $p$-joint spectral radius of $\mathcal{A}$ relative to $\Phi$ and $\mu$ is defined as

$$
\rho_{p}(\mathcal{A})=\limsup _{r \rightarrow \infty}\left(\sum_{\boldsymbol{j} \in \mathbb{Z}_{m}^{r}} \mu_{\boldsymbol{j}}(\Omega)\left\|\boldsymbol{A}_{\boldsymbol{j}}\right\|^{p}\right)^{1 / r p} .
$$

Theorem 4.7. Let $\Phi$ be $\mu$-uniform. Then $\mathcal{A}$ admits an $L_{p}(\Omega, \mu)$ convergent subdivision scheme if and only if the following two conditions hold:

1.

$$
\boldsymbol{A}_{\ell} \mathbf{1}=\mathbf{1}, \quad \ell \in \mathbb{Z}_{m} .
$$

2. The matrices $\boldsymbol{B}_{\ell} \in \mathbb{R}^{n-1 \times n-1}, \ell \in \mathbb{Z}_{m}$, defined by $\boldsymbol{D} \boldsymbol{A}_{\ell}=\boldsymbol{B}_{\ell} \boldsymbol{D}$, satisfy

$$
\rho_{p}(\mathcal{B})<1 \text {. }
$$

Proof. Let us assume that (4.7) and (4.8) are satisfied and show that $\mathcal{A}$ admits a convergent subdivision scheme. To this end, we consider a sequence of vector fields $\boldsymbol{h}_{r}, r \in \mathbb{Z}_{\infty}$, on $\Omega$ defined as

$$
\boldsymbol{h}_{r}^{T}=\frac{1}{n} \mathbf{1}^{T} \boldsymbol{A}^{[r]}
$$

with the convention that $\boldsymbol{A}^{[0]}=\boldsymbol{I}$, the identity matrix. Now, for $r \in \mathbb{Z}_{\infty}$ and $\boldsymbol{c} \in \mathbb{R}^{n}$, using the vector $\boldsymbol{j}^{r}=\left(j_{k}: k \in \mathbb{Z}_{r}\right)$, we obtain the equation

$$
\begin{aligned}
\left\|\left\langle\boldsymbol{c}, \boldsymbol{h}_{r+1}-\boldsymbol{h}_{r}\right\rangle\right\|_{L_{p}(\Omega)}^{p} & =\sum_{\boldsymbol{j} \in \mathbb{Z}_{m}^{r+1}}\left\|\left\langle\boldsymbol{c}, \boldsymbol{h}_{r+1}-\boldsymbol{h}_{r}\right\rangle\right\|_{L_{p}\left(\phi_{\boldsymbol{j}}(\Omega), \mu\right)}^{p} \\
& =\sum_{\boldsymbol{j} \in \mathbb{Z}_{m}^{r+1}}\left\|\left\langle\boldsymbol{c}, \boldsymbol{h}_{r+1} \circ \phi_{\boldsymbol{j}}-\boldsymbol{h}_{r} \circ \phi_{\boldsymbol{j}}\right\rangle\right\|_{L_{p}\left(\Omega, \mu_{j}\right)}^{p} \\
& =\sum_{\boldsymbol{j} \in \mathbb{Z}_{m}^{r+1}}\left\|\left\langle\boldsymbol{c}, \boldsymbol{A}_{\boldsymbol{j}_{r}}^{T}\left(\boldsymbol{A}_{j_{r}}-\boldsymbol{H}\right)^{T} \mathbf{1}\right\rangle\right\|_{L_{p}\left(\Omega, \mu_{j}\right)}^{p} \\
& =\sum_{\boldsymbol{j} \in \mathbb{Z}_{m}^{r+1}}\left\|\left\langle\left(\boldsymbol{A}_{j_{r}}-\boldsymbol{H}\right) \boldsymbol{A}_{\boldsymbol{j}_{r}} \boldsymbol{c}, \mathbf{1}\right\rangle\right\|_{L_{p}\left(\Omega, \mu_{j}\right)}^{p} \\
& =\sum_{\boldsymbol{j} \in \mathbb{Z}_{m}^{r+1}} n^{-p}\left|\left\langle\left(\boldsymbol{A}_{j_{r}}-\boldsymbol{H}\right) \boldsymbol{A}_{\boldsymbol{j}_{r}} \boldsymbol{c}, \mathbf{1}\right\rangle\right|^{p} \mu_{\boldsymbol{j}}(\Omega) .
\end{aligned}
$$

Observe for any $k \in \mathbb{Z}_{m}$ that $\left(\boldsymbol{A}_{k}-\boldsymbol{H}\right) \mathbf{1}=\mathbf{0}$. Thus, there exists a matrix $\boldsymbol{C}_{k} \in$ $\mathbb{R}^{n \times n-1}$ such that $\boldsymbol{A}_{k}-\boldsymbol{H}=\boldsymbol{C}_{k} \boldsymbol{D}$ and we conclude, for $\boldsymbol{j} \in \mathbb{Z}_{m}^{r}$ and $k \in \mathbb{Z}_{m}$, that

$$
\left(\boldsymbol{A}_{k}-\boldsymbol{H}\right) \boldsymbol{A}_{\boldsymbol{j}}=\boldsymbol{C}_{k} \boldsymbol{D} \boldsymbol{A}_{\boldsymbol{j}}=\boldsymbol{C}_{k} \boldsymbol{B}_{\boldsymbol{j}} \boldsymbol{D} .
$$

Consequently there exists a positive constant $\alpha$ such that

$$
\left\|\left\langle\boldsymbol{c}, \boldsymbol{h}_{r+1}-\boldsymbol{h}_{r}\right\rangle\right\|_{p}^{p} \leq \alpha\|\boldsymbol{c}\|^{p} \sum_{\boldsymbol{j} \in \mathbb{Z}_{m}^{r}} \mu_{\boldsymbol{j}}(\Omega)\left\|\boldsymbol{B}_{\boldsymbol{j}}\right\|^{p} .
$$

Thus, for any $\sigma$ such that $\rho_{p}(\mathcal{B})<\sigma<1$ there exists an $r_{0} \in \mathbb{Z}_{\infty}$ and a positive constant $\beta$ such that

$$
\left\|\boldsymbol{h}_{r+1}-\boldsymbol{h}_{r}\right\|_{p} \leq \beta \sigma^{r}, \quad r \geq r_{0} .
$$

Therefore, $\boldsymbol{h}_{r}, r \in \mathbb{Z}_{\infty}$, is a Cauchy sequence in $L_{p}^{n}(\Omega)$ which necessarily converges to some function $\boldsymbol{f} \in L_{p}^{n}(\Omega)$. 
It remains to verify that $\boldsymbol{f}$ is indeed the limit of the subdivision scheme. For this purpose, we observe that, for $r \in \mathbb{Z}_{\infty}$,

$$
\left\|\boldsymbol{D} \boldsymbol{A}^{[r]}\right\|_{p}=\left\|\boldsymbol{B}^{[r]} \boldsymbol{D}\right\|_{p} \leq\|\boldsymbol{D}\|\left\|\boldsymbol{B}^{[r]}\right\|_{p}=\|\boldsymbol{D}\|\left(\sum_{\boldsymbol{j} \in \mathbb{Z}_{m}^{r}} \mu_{\boldsymbol{j}}(\Omega)\left\|\boldsymbol{B}_{\boldsymbol{j}}\right\|^{p}\right)^{1 / p} .
$$

Hence, we conclude that

$$
\lim _{r \rightarrow \infty}\left\|\boldsymbol{D} \boldsymbol{A}^{[r]}\right\|_{p}=0
$$

In other words, we have shown, for any $\boldsymbol{y} \in \mathbb{R}^{n}$ such that $\langle\boldsymbol{y}, \mathbf{1}\rangle=0$, that

$$
\lim _{r \rightarrow \infty} \boldsymbol{y}^{T} \boldsymbol{A}^{[r]}=\boldsymbol{y}^{T}(\mathbf{1} \otimes \boldsymbol{f}) \text {. }
$$

Moreover, 4.9 yields for all $r \in \mathbb{Z}_{\infty}$ that equation (4.13) holds with $\boldsymbol{y}$ replaced by $\mathbf{1}$ and therefore we have confirmed that $\boldsymbol{f}$ is the limit of the subdivision scheme $\mathcal{A}$.

For the converse, we assume that $\mathcal{A}$ admits a convergent subdivision scheme with limit $\boldsymbol{f}$. Then, for any $\boldsymbol{j} \in \mathbb{Z}_{m}^{r}$ and $k, \ell \in \mathbb{Z}_{n}$, we have that

$$
\begin{aligned}
& \mu_{\boldsymbol{j}}(\Omega)\left|\left(\boldsymbol{D} \boldsymbol{A}_{\boldsymbol{j}}\right)_{k, \ell}\right|^{p}=\left\|\left(\boldsymbol{A}_{\boldsymbol{j}}\right)_{k+1, \ell}-\left(\boldsymbol{A}_{\boldsymbol{j}}\right)_{k, \ell}\right\|_{L_{p}\left(\phi_{\boldsymbol{j}}(\Omega), \mu\right)}^{p} \\
& \leq 2^{p}\left(\left\|\left(\boldsymbol{A}_{\boldsymbol{j}}\right)_{k+1, \ell}-f_{\ell}\right\|_{L_{p}\left(\phi_{\boldsymbol{j}}(\Omega)\right)}^{p}+\left\|\left(\boldsymbol{A}_{\boldsymbol{j}}\right)_{k, \ell}-f_{\ell}\right\|_{L_{p}\left(\phi_{\boldsymbol{j}}(\Omega)\right)}^{p}\right) \\
& =2^{p}\left(\left\|\left(\boldsymbol{A}^{[r]}\right)_{k+1, \ell}-f_{\ell}\right\|_{L_{p}\left(\phi_{\boldsymbol{j}}(\Omega)\right)}^{p}+\left\|\left(\boldsymbol{A}^{[r]}\right)_{k, \ell}-f_{\ell}\right\|_{L_{p}\left(\phi_{j}(\Omega)\right)}^{p}\right) .
\end{aligned}
$$

Therefore, there exists a positive constant $\gamma$ such that

$$
\mu_{\boldsymbol{j}}(\Omega)\left\|\boldsymbol{B}_{\boldsymbol{j}} \boldsymbol{D}\right\|^{p} \leq \gamma\left\|\boldsymbol{A}^{[r]}-\mathbf{1} \otimes \boldsymbol{f}\right\|_{L_{p}\left(\phi_{\boldsymbol{j}}(\Omega)\right)}^{p} .
$$

Consequently, summing both sides of this inequality over $\boldsymbol{j}$ and taking into account the fact that $\boldsymbol{D}$ is surjective, we conclude that there is a positive constant $\eta$ such that

$$
\sum_{\boldsymbol{j} \in \mathbb{Z}_{m}^{r}} \mu_{\boldsymbol{j}}(\Omega)\left\|\boldsymbol{B}_{\boldsymbol{j}} \boldsymbol{D}\right\|^{p} \leq \eta\left\|\boldsymbol{A}^{[r]}-\mathbf{1} \otimes \boldsymbol{f}\right\|_{p}^{p}
$$

which proves the claim.

We finally show that a convergent subdivision scheme converges exponentially fast and that its limit enjoys Hölder regularity.

Theorem 4.8. If $\mathcal{A}$ admits a convergent subdivision scheme with limit $\boldsymbol{f}$, then

$$
\lim _{r \rightarrow \infty} \omega_{r}^{1 / r}(\boldsymbol{f}) \leq \rho_{p}(\mathcal{B}), \quad \mu-a . e .
$$

Proof. For any $\sigma$ with $\rho_{p}(\mathcal{B})<\sigma<1$ there exist, by (4.10), numbers $r_{0} \in \mathbb{Z}_{\infty}$ and $\alpha>0$ such that, for $r>r_{0}$,

$$
\left\|\boldsymbol{f}-\boldsymbol{h}_{r}\right\|_{p}=\alpha \sigma^{r} .
$$

Let $\boldsymbol{P}:=\boldsymbol{H}-\boldsymbol{I}$ and observe that $\boldsymbol{P} \mathbf{1}=0$. Hence, there exists a matrix $\boldsymbol{Q} \in$ $\mathbb{R}^{n \times(n-1)}$ such that $\boldsymbol{P}=\boldsymbol{Q D}$. Using (4.9) and (4.11) we find an $r_{1} \in \mathbb{Z}_{\infty}$ and $\beta>0$ such that, for $r \geq r_{1}$,

$$
\left\|\mathbf{1} \otimes \boldsymbol{h}_{r}-\boldsymbol{A}^{[r]}\right\|_{p}=\left\|\boldsymbol{P} \boldsymbol{A}^{[r]}\right\|_{p}=\left\|\boldsymbol{Q D} \boldsymbol{A}^{[r]}\right\|_{p} \leq \beta \sigma^{r}(\mathcal{B}) .
$$


Thus, there is a constant $\gamma>0$ such that, for $r \geq \max \left\{r_{0}, r_{1}\right\}$,

$$
\left\|\mathbf{1} \otimes \boldsymbol{f}-\boldsymbol{A}^{[r]}\right\|_{p} \leq\left\|\mathbf{1} \otimes \boldsymbol{f}-\mathbf{1} \otimes \boldsymbol{h}_{r}\right\|_{p}+\left\|\mathbf{1} \otimes \boldsymbol{h}_{r}-\boldsymbol{A}^{[r]}\right\|_{p} \leq \gamma \sigma^{r} .
$$

Hence, we have, for $r \geq \max \left\{r_{0}, r_{1}\right\}$, that

$$
\omega_{r}(\boldsymbol{f})(y)=\left\|\boldsymbol{f}^{T}(y)\left(\mathbf{1} \otimes \boldsymbol{f}-\boldsymbol{A}^{[r]}\right)\right\|_{p} \leq \gamma \sum_{k \in \mathbb{Z}_{n}}\left|f_{k}(y)\right| \sigma^{r} .
$$

Since $\boldsymbol{f} \in L_{p}(\Omega)$, it is finite $\mu$-a.e. and hence, for $\mu$-a.e. $y \in \Omega$ we conclude that

$$
\lim _{r \rightarrow \infty} \omega_{r}^{1 / r}(\boldsymbol{f})(y) \leq \sigma .
$$

However, $\sigma$ was chosen arbitrarily greater than $\rho_{p}(\mathcal{B})$. This proves the result.

\section{REFERENCES}

1. Z. Chen, C. A. Micchelli, and Yuesheng Xu. A construction of interpolating wavelets on invariant sets. Math. Comp., 68 (1999), 1569-1587. MR 99m:65017]

2. J. E. Hutchinson. Fractals and self similarity. Indiana Univ. Math. J., 30 (1981), 713-747. MR 82h:49026

3. R.-Q. Jia. Subdivision schemes in $l_{p}$ spaces. Advances Comput. Math., 3 (1995), 309-341. MR 96d:65028

4. C. A. Micchelli and H. Prautzsch. Uniform refinement of curves. Linear Algebra Appl., 114 (1989), 841-870. MR 90k:65088

5. C. A. Micchelli, T. Sauer, and Yuesheng Xu. A construction of refinable sets for interpolating wavelets. Results in Mathematics, 34 (1998), 359-372. MR 99h:42063

6. C. A. Micchelli and Yuesheng Xu. Using the matrix refinement equation for the construction of wavelets on invariant sets. Appl. Comp. Harmonic Anal., 1 (1994), 391-401. MR 96k:42044

7. C. A. Micchelli and Yuesheng Xu. Reconstruction and decomposition algorithms for biorthogonal multiwavelets. Multidimensional Systems and Signal Processing, 8 (1997), 31-69. MR 98g:94007

8. C.A. Micchelli. Mathematical Aspects of Geometric Modeling, volume 65 of CBMS-NSF Regional Conference Series in Applied Mathematics. SIAM, 1995. MR 95i:65036

9. K. R. Parthasarathy. Probability Measures on Metric Spaces. Academic Press, 1967. MR 37:2271

Department of Mathematics \& Statistics, State University of New York, The University at Albany, Albany, New York 12222

E-mail address: cam@math.albany.edu,cam@watson.ibm.com

Mathematisches Institut, Universität Erlangen-Nürnberg, Bismarckstr. $1 \frac{1}{2}$, D91054 Erlangen, Germany

E-mail address: sauer@mi.uni-erlangen.de

Department of Mathematics, North Dakota State University, Fargo, North Dakota 58105 and Institute of Mathematics, Chinese Academy of Sciences, Beijing 100080, PeoPLE'S REPUBLIC OF CHINA

E-mail address: xu@hypatia.math.ndsu.nodak.edu 